\title{
Stabilité des digues à talus à carapace en vrac
}

\section{Stability of rubble mound breakwaters with pell mell facings}

\author{
PAR C. BEAUDEVIN
}

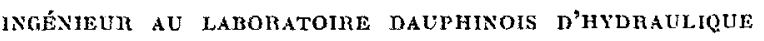

\begin{abstract}
Jusqu'à une dpoque assez récente, la détermination des caracteristiques d'une digue $\dot{a}$ talus ne poubait être qu'empirique. Au cours de ces dernières années, divers autenrs ont proposé des formules permetiant de serrer la réalifé de plus près.

Dans cet espril, les essais relatés ci-après permettent de mienr connaitre tes phenomines lies a lontuque d'une dique à talus par la houle; ane methode pratique de calcul est proposee; enjin, les résnllats des essuis sont contparés à ceur: obtenus, dans d'autres laboratoires, an conrs d'étrdes analognes.
\end{abstract}

\begin{abstract}
Lintil recently it was only possible to determine the characteristics of a rubble mound breakwater empirically. During the last few years several anthors have proposed formulae allowing reality to be approached more closely. With the same end in view the tests described below enables the phenomena related to wave allack on a ribble mound breakwater to be better known. A practical method of calculalion is proposed. Finally the results of the tests are compared with those obtained in other laboratories during similar studies.
\end{abstract}

\section{CONCLUSION DES ETUDES EFFECTUEES AU LABORATOIRE DAUPHINOIS D'HYDRAULIQUE}

\section{I}

\section{Considérations générales sur la stabilité à la houle d'un massif formé d'éléments en vrac}

Quoiqu'il puisse paraître à première vue, il n'est pas facile d'énoncer un critère qui permette de déterminer d'une manière précise la limite de stabilité d'une digue à talus. L’équation personnelle de l'observateur joue en effet Je plus souvent un rôle que l'on ne peut négliger.

Nous avons cherché, au cours des essais relatés dans la présenté communication, à éliminer autant que possible ce facteur subjectif et, dans ce but, nous avons utilisé un critère de stabilité particulier : nous laissions la houle modeler ellemême une figure d'équilibre dans un massif homogène d'éléments en vrac. On vérifiera, au cours d'expériences ultérieures, que le profil ainsi obtenu est stable vis-à-vis des houles d'amplitude inférieure à celle qui l'a modelé, et qu'il est instable (c'est-à-dire qu'il y a chute de blocs) Iorsque l'amplitude de la houle dépasse cette valeur.

Il est done licite d'appeler ce profil « profil d'équilibre $»$ et nous verrons plus loin que, compte tenu d'un coefficient de sécurité convenable, cette notion permet en pratique de déterminer des ouvrages stables. 


\section{II. - Profil d'équilibre caractéristique}

Dans la plupart des cas rencontrés dans la pratique, le profil d'équilibre présente la forme indiquée par la figure 1. Cette forme d'équilibre est assez générale pour que nous l'ayons prise comme référence ef dénommée "forme d'équilibre caractéristique ».

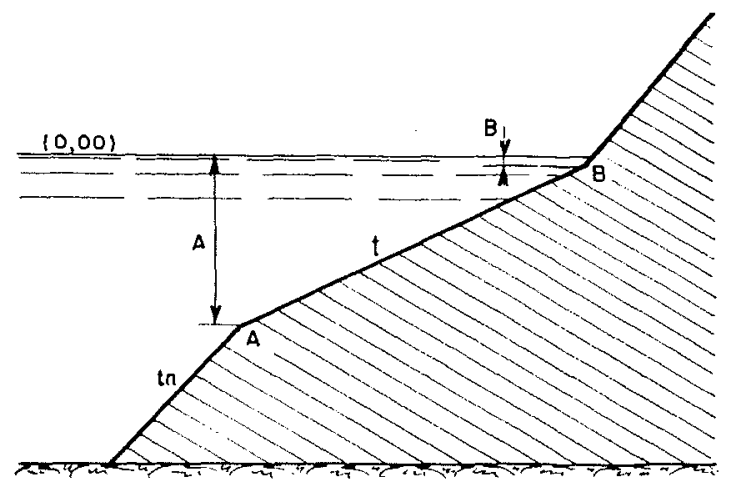

Fig. 1. - Profil d'équilibre caractéristique.

Nous définirons alors l'angle du plateau AB avec l'horizontale, et sa cotangente $t$ respectivement comme « angle d'équilibre » et «talus (ou pente) d'équilibre 》 du matériau donné à la houle considérée.

En dessous du point A, de profondeur A, le talus est sensiblement au talus naturel $t_{n}$ du matériau en eau calme.

Le «plateau» $\mathrm{AB}$ constitue la partie la plus intéressante d'un talus d'équilibre à la houle : il correspond à la carapace d'une digue. Nous allons chercher à déterminer tout d'abord, par des considérations simples, l'influence des divers paramètres sur les valeurs de la pente d'équilibre et sur les cotes $A$ et $B$ du plateau.

\section{III. - Influence de divers facteurs sur la pente d'équilibre à la houle}

\section{Facteurs dimensionnels}

Les facteurs dimensionnels sont : l'amplitude $2 a$ et la période $\mathrm{T}$ de la houle, la profondeur $h$ au pied de l'ouvrage, enfin le poids $P$ et la densité $d$ des blocs composant le massif.

Les études que nous avons effectuées, rejoignant l'expérience classique, montrent que les termes périodes de la houle et profondeur au pied de l'ouvrage agissent seulement commc termes correctifs et qu'on peut, en premic̀re approximation, négliger leur influence. Il restera, par la suite, à les faire intervenir en tant que corrections.

Un calcul élémentaire permet de démontrer que les facteurs dimensionnels autres que la pé- riode et la profondeur interviennent groupés en un nombre :

$$
p=\frac{\mathrm{P}}{(2 a)^{3}} \frac{(d-1)^{3}}{d} .
$$

que nous appellerons "poids réduit 》.

Il reste à définir expérimentalement la forme de la fonction $t=f(p)$ reliant la pente caractéristique au poids réduit, et à déterminer l'influence des termes période et profondeur au pied de l'ouvrage.

INFLUENGE DE LA PÉRIODE. -- Nous avons effectué un certain nombre d'essais destinés à préciser cette influence; nous présentons, figure 2, quelques résultats obtenus en soumettant un massif à des houles de période 1 et $1,8 \mathrm{~s}$.

La conclusion générale de ces essais est qu'une houle est d'autant plus dangereuse que sa période est plus faible, e'est-à-dire qu'elle est plus cambrée. L'influence de la période reste cependant presque toujours faible.

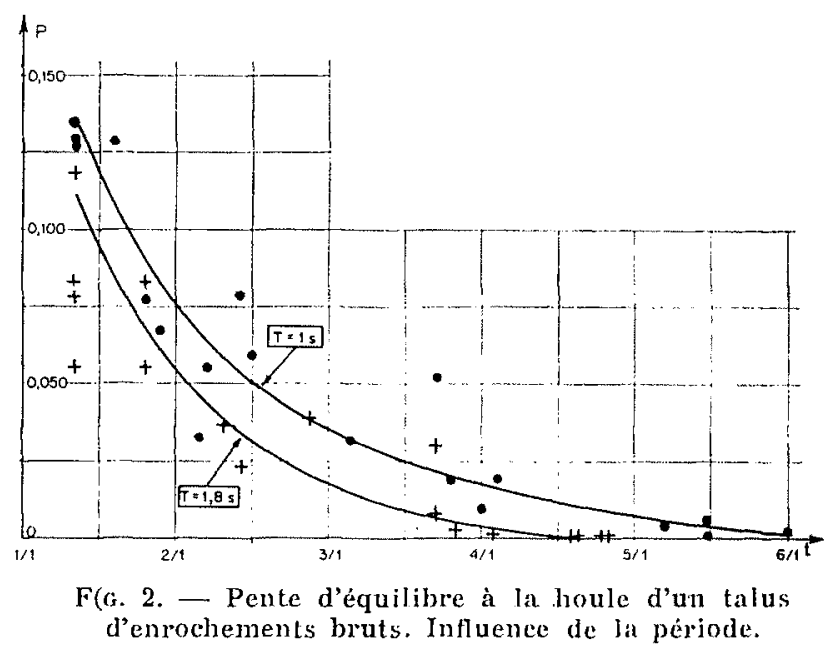

Influence de la profondeur. - Celle influence est complexe car, outre la valeur de la profondeur an pied de l'ouvrage, le profil des fonds au large de celui-ci intervient en effet.

Il semble qu'à condition de prendre en compte l'amplitude de la houle incidente devant l'ouvrage -. et non l'amplitude de la houle au large - l'inlluence de la profondeur reste faible. L'ouvrage est d'autant plus affecté par la houle, à amplitude égale, que la profondeur est plus faible.

\section{FACTEURS NON-DIMLNSIONNLLS}

L'expérience peut seule déterminer l'influcnce exacte de ces paramètres: forme des blocs, profil initial du talus, échelonnement des poids des enrochements constituant la catégorie considérée. 
INFLUENCE DE LA FORME DES BLOCS. - - Au cours de nos essais, nous avons successivement considéré différentes formes de blocs : blocs cubiques à arêtes vives, bloes cubiques à arôtes émoussées, blocs arrondis (assez proche de sphèes), enrochements à arêtes vires, enrochements à arêtes émoussées, tétraèdres, enfin sphères.

Les essais ont montré que les coubes $p=f(t)$ relatives à ces divers bloes se déduisaient sensiblement les unes des autres par affinitć. Il est ainsi possible de caractériser chaque forme de blocs par un « facteur de forme 》 constant.

On peut ainsi dire que, toutes choses égales par ailleurs, un enrochement émoussé de 1 tonne possède une stabilité équivalente à celle d'un enrochement brut de carriere de $750 \mathrm{~kg}$ ou d'un bloc cubique émoussé de $500 \mathrm{~kg}$ ou enfin d'un bloc cubique brut de décofrage de $350 \mathrm{~kg}$.

Le fait que les courbes de stabilité relatives aux divers blocs considérés peuvent sensiblement se déduire les unes des autres par affinité, résulte d'une constatation expérimentale ef cetle conclusion se vérifie dans la quasi-totalité des cas de la pratique. Nous mentionnerons cependant une exception : il s'agit des blocs artificiels tétrapodes. Ceux-ci possèdent la curieuse propriété d'offrir une stabilité sensiblement équivalente aux pentes douces et aux pentes raides. Ce lait provient essentiellement du chargement réciproque des bloes qui se produit aux pentes raides.

Il en résulte qu'il y a intérêt en général à employer les tétrapodes en carapace en perte mide, de l'ordre de $4 / 3$, par exemple. Pour la pente $4 / 3$, toutes choses égales par ailleurs en particulier lusure des blocs, il est sensiblement équivalent d'employer des tétrapodes de poids $P$, des blocs cubiques de poids $3 \mathrm{P}$ ou des enrochement de poids $6 \mathrm{P}$.

INFIIENCF DE L'ECHELONNEMLNT DES POIDS A I'INTHRTELR D'TNE CATÉGORIE D'ENROCHEMENTS. Ce facteur intervient lorsqu'il s'agil de définir le poids earaeléristique $P$ de la catégorie.

Nos essais nous ont amenés aux conclusions suivantes :

En regle générale, un mólange d'enrochements de diverses tailles possède un poids caractérislique égal ou supérieur au poids moyen des enrochements. Dans certains cas, on peut même dire qu'un melange d'enrochements peut ètre équivalent aux bloes les plus lourds qui le composent. Ce fait peut s'expliquer facilement: lors de latlature du lalus par la houle, les blocs légers placés entre les blocs plus lourds trourent ainsi des logements qui leur ofrrent une certaine protection contre la houle en mome temps que des appuis efficaces. On concoit done que la stabilité de l'ansemble des enrochements puisse être cquivalente a celle des blocs les plus lourds.
On peut en conséquence admettre que le poids caractéristique intervenant dans le calcul de la slabilité est le poids moyen de la catégorie considérée. Dans la grande majorité des cas que l'on peut être amené à rencontrer, ceci revient à prendre une certaine marge de sécurité.

\section{IV. - Influence des divers facteurs sur les cotes $A$ et $B$ du plateau}

\section{Cote de l'extrêmité inférieure A du plateau}

INFLUENCE DE L'AMPLITUde DE LA HOULE. Lorsqu'une houle d'amplitude lentement croissante aborde un talus, il n'y a d'abord pas d'allaque. Puis, pour une certaine valeur de l'amplitude, il apparait un palier $A B$ par chute des enrochements (fig. 1). La cote de l'extrémité inférieure $A$ du plateau est alors sensiblement proportionnelle à l'amplitude de la houle. Si celle-ci s'accroil encore on constate, à partir d'une certaine valeur, que le talus présente une double cassure, le point A se dédoublant (fig. 3) en

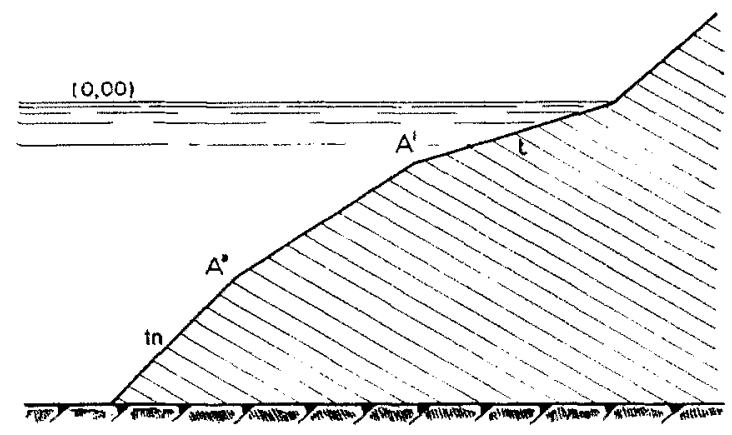

Frg. 3. - Talus à deux ruptures de pente.

deux points $A^{\prime}$ et $A^{\prime \prime}$. Si l'amplitude augmente cneore, $A^{\prime}$ reste fixe cependant que la profondeur de $A^{\prime \prime}$ continue à croître, sensiblement proportionnellement à l'amplitude.

INILUENCE DE LA PÉRIODE DE LA HOULE. - Lorsqu'il n'existe qu'une seule cassure, sa profondeur $A$ est sensiblement indépendante de la période. Lorsqu'il existe deux cassures, la cote de la plus profonde $A^{\prime \prime}$ est également sensiblement indépendante de la période, cependant que la cote de la cassure supérieure $\mathrm{A}^{\prime}$ en dépend.

INFLTFNCE DFS CARACTÉRISTIQUES DES BLOCS (Poms, DFNSITÉ IN Forme). - Ce paragrapho nous conduit à une conclusion trìs surprenante: les essais montrent en effet que la cote A de l'extrémité inférieure du plateau est pratiquement indépendante des caractéristiques des blocs et n'est fonction que de lamplitude de la houle. Nous arons vu que A etait proportionnel à l'am- 
plitude; le terme de proportionnalité ne varie que fort peu avec les caractéristiques des blocs.

Au cours de nos essais où le poids réduit a varié de 1 à 200 (en valeur relative), le facteur de proportionnalité reliant $A$ à l'amplitude a varié entre les valeurs extrêmes 1,6 et 1,2 (valeur moyenne 1,3 ).

Il peut paraître surprenant que la cole du point A à partir duquel les enrochements ne sont plus remués par la houle soit pratiquement indépendante des caractéristiques de ces enrochements. Ce fait est contraire aux règles couramment utilisées en travaux maritimes. Signalons cependant qu'avant nous, le brigadier BAGNold avait observé un phénomène analogue au cours des essais de stabilité des plages qu'il effectua sur modèle réduit. Il remarqua, en effet, que la profondeur à laquelle les galets ne sont pas susceptibles d'être déplacés par la houle est proportionnelle à l'amplitude de celle-ci et indépendante du diamètre des galets.

Le facteur de proportionnalité reliant cette profondeur à l'amplitude de la houle était de l'ordre de 1,1 à 1,4 ce qui rejoint bien les conclusions de nos essais en étendant encore leur domaine de validité.

INFLIEYCE DE LA PROFONDEUR $h$. Celle-ci scmble faible.

\section{Cote de l'extrémité supérieure $B$ du plateau}

Ia limite supérieure $B$ du plateau $A B$ est toujours proche du $(0,00)$ quoiqu'en général très légèrement plus bas.

\section{V. - Divers domaines définis au cours de cette étude}

DOMAINE DES DIGUES ET DOMAINE DES PLAGES. -Lorsque le poids réduit est inférieur à une cerlaine valeur, de l'ordre de $2.10^{-3}$, le profil d'équilibre n'est plus le profil d'équilibre caractéristique de la figure 2. Il peut alor's prendre des formes plus complexes.

Cette valeur $p=2.10^{-3}$ est obtenue, par exemple, pour des blocs de densité 2,8 de poids $1 \mathrm{~kg}$, soumis à une houle d'amplitude 1 motre.

Ces chifres correspondent à une plage de gros galets. On voit que la condition $p=2.10^{-3}$ marque sensiblement une limite entre les digues à talus et les plages de galels. Notre élude s'est donc limitce à cette valeur inférieure du poids réduit, au-delà de laquelle la notion simple de pente d'équilibre devient insulfisante.
STABILITÉ TOTale ET STABILITÉ STATISTIQUE. A l'intérieur du domaine des digues à talus peuvent ètre distingués deux modes de stabilité des blocs à la surface du talus, l'équilibre une fois établi :

- soit les blocs sont individuellement stables : on a stabilité lotale»,

- soil chacun d'entre eux peut être animé de mouvements alternatifs, autour d'une position moyenne et ceci sans que le profil du massif évolue si la houle l'attaque normalement : on a ce que l'on peut appeler «stabilité statistique».

Ces deux domaines soni séparés par une valeur du poids réduit voisine de $p_{0}=22.10^{-3}$ pour des enrochements bruts de carrière.

Pour des enrochements émoussés, $\mu_{0}=30.10^{-2}$.

On a donc stabilité totale lorsque $\mu>p_{0}$ et stabilité statistique lorsque $p<p_{0}$.

Il est possible de tracer des abaques généraux concrétisant les divers résullats exposés jusqu'ici. Nous en donnerons comme exemple la figure 4, relative à des enrochements émoussés sans coefficient de sécurité.

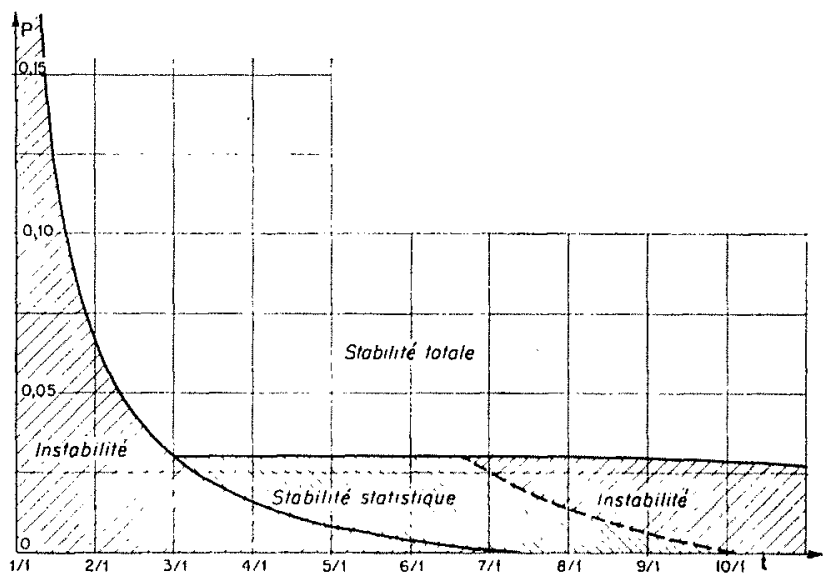

Fia, 4. - Stabilité a la houle d'un massif formé d'éléments en vrac.

Signalons enfin que nos essais onl permis de mettre en évidence l'existence d'une zone d'instabilité relative aux pentes trop douces composées de blocs trop légers. De tels talus sont remaniés par la houle, les éléments étant transporlés vers le haul, cependant que la pente se raidit. L'évolution conduit a un talus plus raide que le talus initial, et représenté approximalivement par la courbe en tirets. 


\section{DEUXIEME PARTIE}

\section{AVANT-PROJET D'UNE DIGUE A TALUS A CARAPACE EN VRAC}

Les digues a talus a carapace en vrac que lon est amene à réaliser en pratique différent, parfois notablement, de l'ouvrage schématique que nous avons considéré jusqu'ici. On peut cependant, moyeunant certaines précautions, utiliser les résultats précédents pour le tracé de l'avant-projet d'une digue à talus.

\section{DETERMINATION DE LA PENTE DE LA CARAPACE}

Les essais que nous avons effectués permettent de déterminer la relation entre le poids réduit des éléments de carapace et la pente de celle-ci. Il reste cependant à définir le coefficient de sécuvile dont il fant affecter ce poids réduit pour obtenir des garmaties suffisantes de stabilité. Ce coeffieient est assez deve pour de multiples raisons. Il est à fixer par la pratique des travaux à la mer; il nous semble cependant que la valeur 2,5 (par rapport aux résultats bruts des essais) ofre wne sécurite convenable dans la majorité des eas.

Compte tenu de ce coefficient de serurite 2.5, et consideranl des hloes légèrement imousses, nu peut donner la formule pratique suivante ${ }^{*}$ :

$$
\mathrm{P}=\mathrm{K}(2 a)^{3} \frac{d}{(d-1)}\left(\frac{1}{-0,8}-0,15\right)
$$

$\pi=0.25$ pour des enrochements,

$$
\mathrm{K}=0,12 \text { pour des boes cubiques. }
$$

Cotte formule est valable pour toutes les leurs du poids reduit supezienres $4.10^{-\frac{3}{3}}$; elle convient par consequent dans la quasi-totalite thes cas qua l'on peut renconirer en rẻaliz. Elle ne pretend que condenser de facon aussi simple que possible les risultats experimentaux et n'a pas de visces theoriques.

L amplitude 2 a intervenant dans la formule est lampotitude lacale de la houle ì l'emplace-

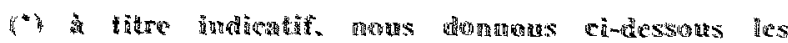

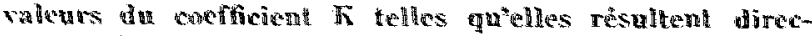

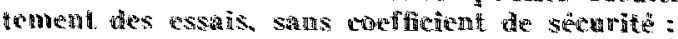

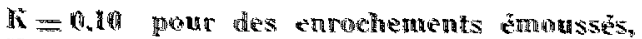

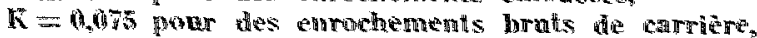

$\mathrm{K}=0.050$ pora des blocs cubionues èmmassés.

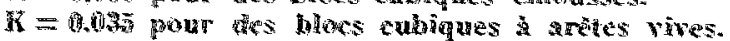

ment de l'ouvrage. Elle peut être déterminée par des mesures directes, ou par l'emploi conjugué des abaques de prévision de houle et d'un plan de vagues.

Le poids $P$ figurant dans la formule est le poids moyen (moyenne arithmétique) des enrochements formant la carapace ou le poids individuel des blocs cubiques. Enfin $t$ est la cotangente de l'angle que forme, avec l'horizontale, la pente du talus.

Le poids donné par la formule (1) assure la stabilité statistique, c'est-à-dire que la pente $t$ demeure inchangée, bien que les enrochements puissent être animés, à la surface du talus, de mouvements alternatifs si la valeur du poids réduit est trop petite. La stabilité tofale, nécessaire pour éviter l'érosion de l'ouvrage par me houle oblique, sera assurée par la condition supplémentaire :

$$
\mathrm{P}>\mathrm{K}^{\prime}(2 a)^{3} \frac{d}{(d-1)^{3}}
$$

$$
K^{\prime}=0,030 \text { poux des enrochements. }
$$

Cette valeur de $K^{\prime}$ ne comprend pas de coefficient de sécurité; celui-ci est à déterminer dans chaque cas particulier, en fonction de la graviti des attaques par des houles obliques.

\section{DÉTERMINATION DE LA COTE INFÉRIEURF. DE LA GARAPACE}

On peut conclure de nos essais que, du strict point de vue résistance à la houle, louvrage peut ofre constitue, sous la cote $(-1,3 \times 2 a)$, par des bloes relativement lögers, réglés à leur talus nalurel d'équilibre en eau calme, sans que l'on ait v craindre d'érosions.

Cette valeur ne comprend pas de coefficient de sereurité, il est donc indiqué de tenir compte de Faction de la houle jusqu'à une profondeur quelque peu superieure, par exemple 1.5 fois lamplitude pour fixer les idees.

11 n'est cependant en général pas néessaire de conduire la carapace elle-mène jusquà la cote $(-1,5 \times 2 a)$. ear on peut, à sa partie inférieure. remplacer les lourds eléments de carapace par des blors plus légers. Il s'agit alors de cas despee qu'il est nécessaire de prëciser par une elude particuliere sur modele réduit. 


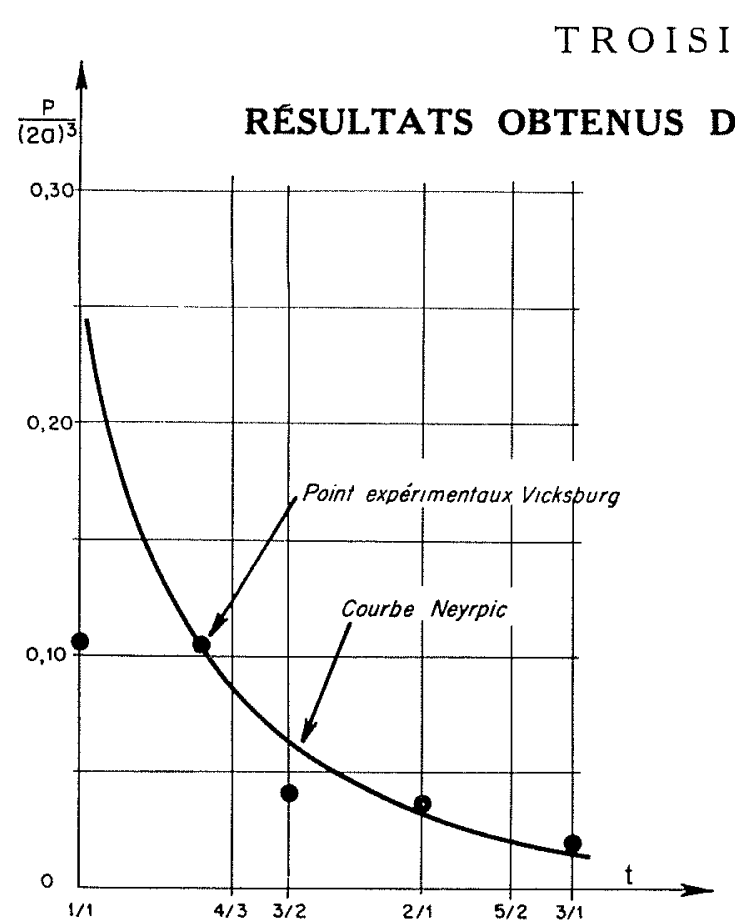

Fig. 5. - Résultats d'essais effectués à Vicksburg. Enrochements émoussés. Critère de « léger dommage ».

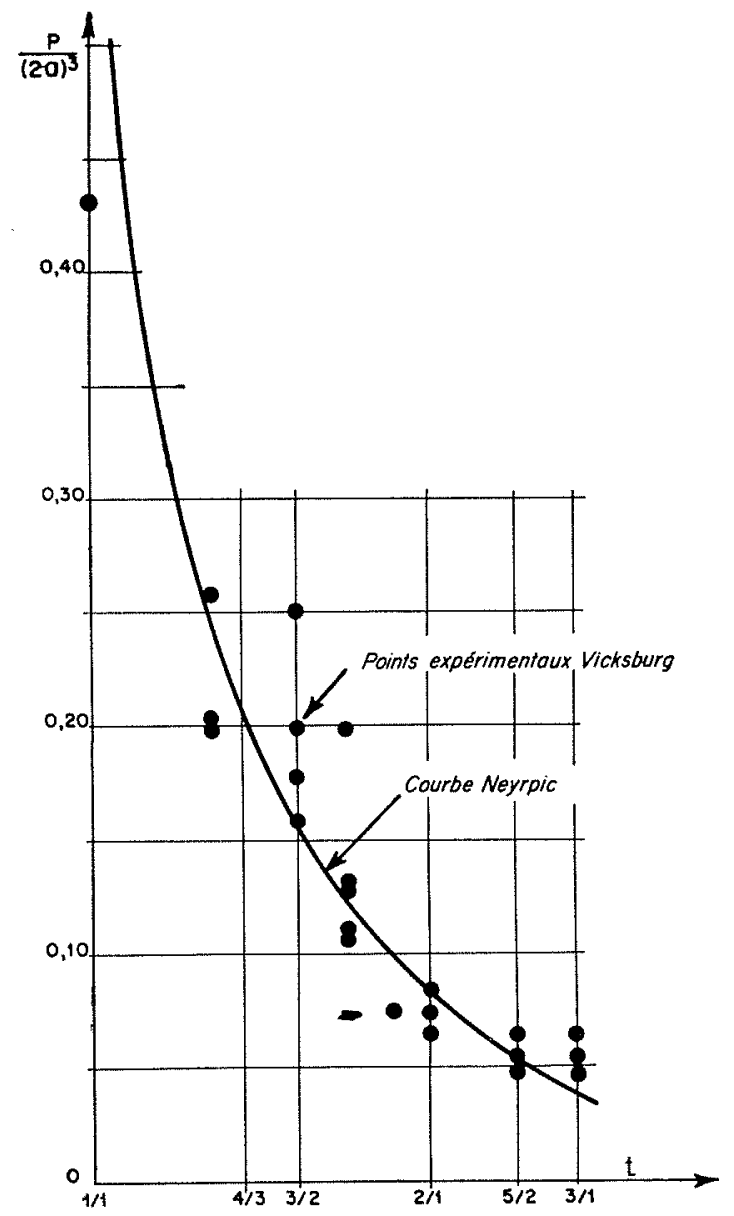

Fig. 6. - Résultats d'essais effectués à Vicksburg. Enrochements émoussés. Critère de «non-dommage».
On peut citer un certain nombre d'études effectuées dans divers laboraloires, relatives à la détermination des digues à talus. A notre comnaissance, toutefois, il n'existe qu'une seule étude assez systématique de ce problème. Elle fut effectuée en 1953 au «Waterways Experiment Station, de Vicksburg ».

Les modalités de cette étude diffèrent notablement de celles de nos essais. La comparaison que l'on peut faire entre les résultats dégagés par les, deux laboratoires n'en est que plus intéressante.

Les expérimentateurs américains ont été ame-

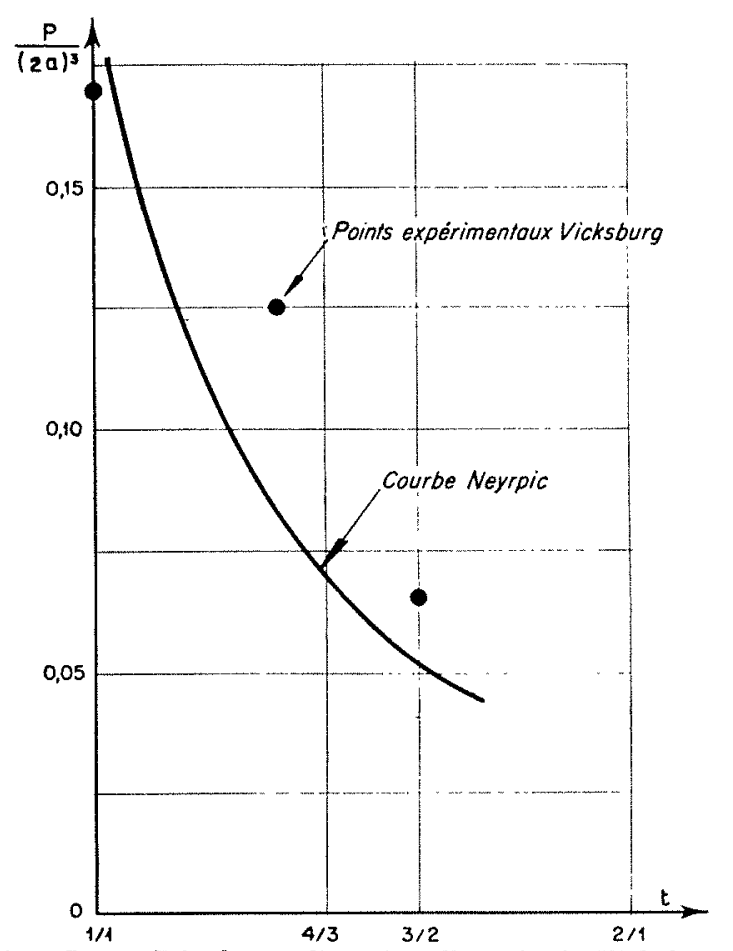

Fig. 7. - Résultats d'essais effectués a Vicksburg.

Blocs cubiques bruts. Critère de «non-dommage ».

nés à définir deux critères de stabilité. Le premier, dit « critère de non-dommage», conduit à des ouvrages offrant toute garantie de stabilité. Il correspond donc à la formule pratique donnée plus haut.

Le second, dit « critère de léger dommage», correspond à un ouvrage dont la stabilité à la houle est limite. Il correspond donc aux résullats bruts de nos essais, sans coefficient de sécurité.

Les points déduits des expériences américaines doivent donc se placer au voisinage des courbes résultant de nos essais. On voit sur les figures 5,6 et 7 qu'il en est bien ainsi et que l'accord 
peut être considéré en général comme satisfaisant.

Mais l'étude du laboratoire de Vicksburg offre d'atres conclusions intéressantes. En particulier, ello a permis de constater que les bloes arti- ficiels cubiques possédaient une stabilité supérieure a celle d'enrochements naturels de même poids et densité. La comparaison des figures précédenles illustre bien celle conclusion, qui est egalement colle de nos essais.

\section{CONCLUSION}

Nous voudrions, pour conclure, revenir sur quelques points particuliers que nos études ont pu mettre en bridence et qu'il nous semble intéressant de souligner.

1 " Le talus naturel, voisin de $4 / 3$, est stable vis-à-vis de houles d'amplitude non négligeable dautant plus fortes que le poids des éléments est plus grand $\left.{ }^{*}\right)$.

2" Une houle atlaque d'autant plus durement um talus, à amplitude cigale, que sa période esl plus faible. Les houles les plus cambrées sont done les plus dangereuses pour la carapace d'un auvrage.

3" Les blocs artificiels cubiques sont sensiblement équivalents à des enrochements de poids double et d'usure comparable. II apparait done que les bloes artificiels en vrae possèdent une stabilitẻ supérieure à celle des enrochements naturels $(* *)$.

4" La profondeur limite a'action d'une houle, sur une catégorie d'enrochements, c'est-à-dire la profondeur jusquài laquelle il est possible de monter un talus naturel formé de ces enroche- ments sans constater d'érosion, dépend peu dı poids des enrochements et n'est fonction pratiquement que de l'amplitude de la houle.

Nous tenions à attirer l'attention sur ces quelques points, qui ne sont pas toujotirs conformes aux idées genéralement admises.

(*) Ces conclusions rejoignent bien eelles que M. l'Ingénicur Surleat, Chet d'Exploitation du port de Casablanca, expose dans un récent numéro de la revuc Travaux. Il note en cffet que la carapace de la jetée Delure, formée de blocs artificiels de $100 \mathrm{~T}$ et réglée à la pente $1 / 1$, résiste sans adoucissement aux fortes houles qu'elle subit.

(**) Ce point particulier méritait d'etre mis en éridence. Nous n'en eiterons comme application que le brise-lames Est de Mers-el-Kébir où des blocs cubiques en vac posés à la pente $2 / 1$ sont mis en place pour résister à des houles de 8 mètres d'amplitude. Le poids de ces blocs a été déterminé par des études sur modèle réduit : il est de $40 \mathrm{~T}$. Appliqué à ce cas particulier, notre formule domnerait un chiffre de $36 \mathrm{~T}$. Nous signalerons que des blocs cubiques de $27 \mathrm{~T}$ posés sur cet ouvage selon la pente $2 / 1$ ont, au mois d'arril 1954 , subi sans dommages une houle de plus de $7 \mathrm{~m}$ d'amplitude.

\section{DISCUSSION}

Président: M. WAm

M. Miche remarqua que in cente $-1,3 \times 2$ a au-dessus

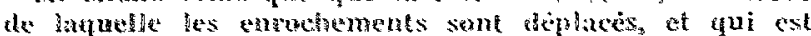
par conseguent fonction de lomplitude de la houle, doit atre en relation direwe ave le dobut du deferlement sur le talus ce qui pourrait are la raison de la table influence sur cette profondeur d"instabilite, de la densite et de la forme des enrochements.

M. Hesumbix pense quetfectivement la sllabilite du talus au-dessous de cette protondeur indinue wne diffé-

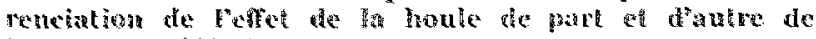
la coler du detevlement.

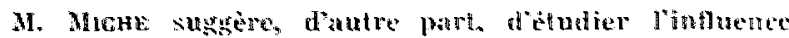

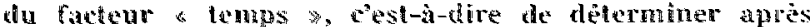
combirem de cyeles de houle l'etat limite de stabilite

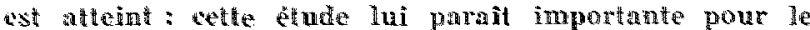

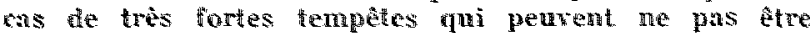

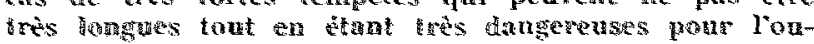
vrase: ar, les recherelnes de nombreax daboratoires, qui coneduent, comme celles rapportées par M. BEalidevix a l'indépendance de la periode ne paraissent eoncernes que litat limite, c'est-a-dire celui atteint après un assez grand nombre de cycles de houle.

M. Brafoeron indique que, pour des études particulières. te Laboratoire Datphinois d'Hydualique a l'habitude de considerer laction de la houle pou un temps limite, qui introduit un parametre de plus, dont léfude systrmatique qu'il a expose avait precisément pour bat de se liberer en ne considerant que lequilibre limile a bout diu temps suffisamment grand. Il ajoute que, hans l'etude du Lahoutoire americail de Vicks burs, les auteurs ont verifie que la similitude sappliquait a cet etat conulibre provisoire.

Répondant deux questions de M. River, M. BEArDESIN indique :

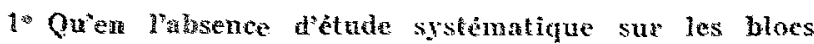
aroimes jointits, on pent cependant conclure 
d'études particulières que la stabilité d'un ouvrage ainsi conçu et pour lequel les conditions d'appui et l'influence des sous-pressions ont été soigneusement déterminées, nécessite des blocs de poids moindre que les carapaces en vrac, mais que celles-ci sont moins fragiles et plus facilement rechargeables après une tempète.

$2^{\circ}$ Que, dans les mers à marée, on peut établir un profil qui répond dans sa partie supérieme aux conditions de stabilité à marée haute et, dans sa partic infërieure, aux mèmes conditions à marée basse.

M. Rioer estime cependant qu'il peut parfois n'être pas facile de concilier les deux conditions.

Répondant à une question de M. Lazard, M. Beaunevin indique que les essais particuliers de carapace en vrac comportent toujours deux couches de blocs, que les essais exposés ici, effectués en canal vitré, ont porté sur des massifs homogènes, mais que leurs. résultats relatit's à la carapace sont applicables aux ouvrages in situ dont les noyaux sont constitués de blocs tout venants, d'après les études du Laboratoire de Vicksburg.

M. Le Président précise que de tels ouvrages nécescitent, entre tout venant et carapace, des couches intermédiaires dont il faudrait aussi déterminer la granulométrie pour aller jusqu'au fond du problème.

M. Beaudevin estime que cette granulométrie est fixée par une condition de filtre - il faut civiter que les petits enrochements ne filtrent à travers les interstices des grands qui les reconvrent - et par une condition de stabilité dans les phases de la construction.

M. Mrche constate que les résultats exposés par M. BEAUdevin autoriseraient a priori, pour les digues à talus, l'emploi de remblais de sable à 10 mètres de profondeur pour des houles allant jusqu’à $7 \mathrm{~m}$ de creux. Or, pratiquement, on n'utilise pas dans ces conditions de remblais de sable à moins de 25 mètres et de petits enrochements à moins de 15 mètres de profondeur. Il semble donc qu'il $y$ ait, en dehors de la stabilité apparente ou « statistique » de la surface du talus, certains facteurs d'instabilité avec le temps, tels que l'aspiration des matériaux fins. En tout cas, ceci mériterait d'être élucidé, car si ces résultats indiqués étaient définitivement confirmés, on pourrait étre conduit à des économies substantielles dans l'exécution des ouvrages.

M. Beaudevin fait remarquer que ces écoriomies, portant sur des matériaux en général peu cốteux, doivent rester assez limitées, d'autant plus qu'elles doivent laisser subsister une marge de sécurité suffisante. Par ailleurs, ainsi qu'il a été précisé au cours de l'exposé, le domaine cxploré au cours des essais est limité aux matériaux de la classe du tout-venant et on ne peut extrapoler les résultats à des matériaux plus petits, sable par exemple.
M. Fonest signale la possibilitẻ d'attribuer les incertitudes des résultats à un effet d'échelle sur le mode de déplacement des matériaux sous l'effet de la houle, observé au'Laboratoire National d'Hydraulique: les échelles des essais du Laboratoire Dauphinois d'Hydraulique sur lesquels a porté l'élude systématique de i. Beaudevin évoluant entre $1 / 25$ et $1 / 100$, il est possible que, pour un mìne profil, les matériaux se déplacent par roulement dans les modèles au $1 / 50$ et par saltation au $1 / 25$.

Le fait, que dans les expériences de M. Beaudevin, les phénomènes de saltation aient été diminués par suite de l'effet d'échelle, explique les grandes variations, en fonction de leur forme, du poids des bloes à stabilite égale. joue un rôle bien plus important pour le roulement que pour la saltation.

Pour passer aux phénomènes naturels, il $\mathrm{y}$ a donc lieu de resserrer la gamme des poids des blocs à stabilite égale et de ne pas attribuer une trop grande importance aux possibilités d'encastrement des blocs.

M. Beaudevin estime que cet effet d'échelle doit être négligeable.

M. Devineux rappelle que, dans une communication au Comité Technique de la S.H.F. concernant l'étude générale du déferlement de la houle, effectuée au Laboratoire Central d'Hydraulique de France, à Maisons-Alfort en 1949-1950, M. Lanras a signalé l'apparition d'un cerlain effet d'échelle, lorsque la période de la lonule en canal est inférieure à $3 \mathrm{~s}$ : pour une valeur dombe de l'ampljtude de la houle au large, la houle en canal déterle par profondeur plus faible que dans la réalité; pat ailleurs, à amplitude égale au point de déferlement, la houle en canal déferle par profondeur plus lorte que dans la nature. Cet effet ne devient sensible que lorsque la période de la houle descend au-dessous de 2 s. Au-dessus de cette valeur, il est faible et ne permettrait de jeter un doute sur la validité des expériences.

M. Braudeau se demande si cet effet d'échelle ne provient pas de la différence des perméabilités du modèle et de l'ouvrage en place.

M. Beaunevin croit qu'il y a en somme une double cause de la différence entre les résultats du modèle el le comportement de l'ouviage :

$1^{\circ}$ La différence entre le modèle homogène et l'ouvrage forme d'une carapace et d'un noyau de granulométrie différentes;

$2^{\circ}$ La différence des perméabilités à la houle; cette dernière cause fait l'objet d'études en cours au Laboratoire Dauphinois d'Hydraulique desquelles on peut, dès maintenant, conclure qu'il faut construire la carapace de l'ouvrage réel avec des éléments plus gros que ceux que domerait l'application de la similitude de Froude, mais que ce grossissement est inutile sous la carapace.

M. Je Président remercic M. Beaudevin. 